\title{
Fixed Time and Fixed Angle External Fixation in the Treatment of Gartland Type III Supracondylar Humerous Fractures in Children
}

\author{
Wei Cai ${ }^{*}$, Yang Wenbin, Liao Hailang, Zeng Lingyuan \\ Department of Orthopedics, People's Hospital of Laibin, Laibin, China \\ Email address: \\ wei13317828080@163.com (Wei Cai) \\ ${ }^{*}$ Corresponding author
}

\section{To cite this article:}

Wei Cai, Yang Wenbin, Liao Hailang, Zeng Lingyuan. Open reduction Internal Fixation Combined with Fixed Time and Fixed Angle External Fixation in the Treatment of Gartland Type III Supracondylar Humerous Fractures in Children. International Journal of Clinical and Experimental Medical Sciences. Vol. 4, No. 4, 2018, pp. 57-62. doi: 10.11648/j.ijcems.20180404.11

Received: August 15, 2018; Accepted: September 14, 2018; Published: October 12, 2018

\begin{abstract}
Objective: To assess the efficacy of open reduction internal fixation (ORIF) combined with fixed-time and fixedangle external fixation (FTFAEF) in the treatment of Gartland type III supracondylar humerous fracture (SHF) in children. Methods: Clinical data of 172 children with Gartland type III SHF from March 2012 to December 2017 were prospectively analyzed. All 172 patients were initially treated with ORIF, then 86 underwent FTFAEF for 3 weeks post-surgery (intervention group) while the remaining 86 received conventional plaster external fixation $\left(70^{\circ}-90^{\circ}\right)$ for 3 weeks post-surgery (control group). The plaster casts were removed from both groups after the 3-week fixation period and functional exercise was initiated. Regular clinical and radiologic follow-ups were conducted on all patients. Range of motion (ROM) measurements and modified Hospital for Special Surgery (HSS) elbow function assessments were performed at 1 and 3 months post-surgery. Results: At 1 month post-surgery, both ROM and modified HSS scores were significantly higher in the intervention group $\left(85.8 \pm 6.1^{\circ}\right.$ and $65.2 \pm 3.6^{\circ}$, respectively) than in the control group $\left(62.3 \pm 5.2^{\circ}\right.$ and $56.6 \pm 2.1^{\circ}$, respectively)(both $\left.\mathrm{P}<0.05\right)$. After 3 months, both ROM and modified HSS scores were still significantly higher in the intervention group (132.0 $\pm 4.7^{\circ}$ and $98.5 \pm 1.3^{\circ}$, respectively) than in the control group $\left(107.5 \pm 24.4^{\circ}\right.$ and $85.0 \pm 10.3^{\circ}$, respectively) (both $\left.\mathrm{P}<0.05\right)$. Furthermore, the percentage of patients scoring excellent on the HSS scale was significantly higher in the intervention group (100\%) than in the control group $(74.42 \%)(\mathrm{P}<0.05)$. Conclusions: ORIF combined with FTFAEF is an efficacious approach that should be widely promoted for the treatment of Gartland type III SHF in children.
\end{abstract}

Keywords: Supracondylar Humerous Fracture, Children, Open Reduction Internal Fixationn, Dysfunction, External Fixation

\section{Introduction}

Supracondylar humerus fractures (SHF) are common elbow fractures in children, accounting for $30 \%-40 \%$ of elbow fractures. In some of these severe fractures, such as Gartland type III supracondylar humerus fractures, the clinical symptoms are large displacement of the fracture end, unstable fracture end, and severe soft tissue injury around a joint, which can cause elbow-joint dysfunction if not handled properly. Therefore, such fractures require internal fixation for treatment. After the operation of the supracondylar humerus fracture, the elbow is usually immobilized by plaster cast at a flexion angle of $70^{\circ}$ to $90^{\circ}$ for three to four weeks. Cast immobilization at a fixed angle for a long time can cause elbow-joint dysfunction. The longer the plastercast remains, the more pronounced the elbow-joint dysfunction. Elbow organized hematoma and soft tissue contracture are the major causes of elbow-joint dysfunction following an operation of the supracondylar humerus fracture. The characteristics of soft tissue are elasticity and plasticity. In the process of repairing the elbow injury, the stress generated during the extending and bending of the elbow joint can 
lengthen the soft tissue around the joint. When the stress is removed, the lengthened soft tissue can return to the original length, due to the elastic deformation of the soft tissue. Sometimes, when the stress is removed, the lengthened soft tissue can maintain a lengthened condition, known as the plastic deformation of the soft tissue. Whether the soft tissue presents elastic deformation or plastic deformation depends on the magnitude and duration of the stress. In order to ensure the stability of the fracture end, improper extending and bending of the elbow joint is prohibited after the operation. However, the single angle of immobilization increases the risk of elbow-joint dysfunction. To address this problem, our hospital applied open reduction internal fixation (ORIF)surgery combined with fixed-time and fixed-angle external fixation(FTFAEF) to treat Gartland type III supracondylar humerus fractures in children from March 2012 to December 2017 and obtained promising results.

\section{Materials and Methods}

\subsection{General Information}

Inclusion criteria: 1) Aged 3.5-12 years, normal intelligence with a certain level of linguistic competence; 2) Closed Gartland type III SFH; 3) Absence of significant vascular nerve injury in the affected arm; 4) Absence of head injury or brain disease; 5) Surgery performed 3d-7d after injury; 6) Patients available for follow-up after at least 3 months.

Patients who met the inclusion criteria were randomly assigned into an intervention $(n=86)$ or control group $(n=86)$. Intervention group: 56 males, 30 females, $8.4 \pm 2.1$ years of age, with $4.8 \pm 1.5 \mathrm{~d}$ between injury and surgery. Control group: 50 males, 36 females, $7.9 \pm 2.6$ years of age, with $5.1 \pm 1.7 \mathrm{~d}$ between injury and surgery. There were no significant differences in patient age and time between injury and surgery between the two groups $(\mathrm{P}>0.05)$. This study was approved by the Ethics Committee of the hospital. All patients and their families provided informed consent.

\subsection{Materials}

Functional plaster cast: An orthopedic splint was cut into two segments based on the lengths of the patient's upper arm and forearm. Metal hinge plates were inserted into one end of both splints and connected to form a hinge joint. The splints were tied onto both the patient's upper arm and forearm, molded to the shape of the arm and allowed to harden.

\subsection{Treatment}

\subsubsection{Surgery}

Patients were placed in a supine position under general anesthesia or a brachial plexus block. After the entire arm was disinfected and covered with a surgical drape, closed reduction of the fracture was firstly performed[1, 2]. If this procedure failed, a lateral approach was used. Briefly, a 2$4 \mathrm{~cm}$ incision was created lateral to the distal humerous, and the tissues between the biceps and triceps were incised and separated until the fractured ends of the bone were exposed. After limited peeling of the periosteum and removal of the soft tissues and blood clots at the ends of the fracture, the bones were realigned and fixed by inserting two $1.5 \mathrm{~mm}$ - or 2.0mm-diameter Kirschner needles (K-needles) through the lateral and medial epicondyles until $2.0 \mathrm{~mm}$ of the needle has pierced through the cortical bone of the proximal humerous. The patient's elbow was moved to confirm joint movement, and if satisfactory, the distal ends of the K-needles were bent, cut then embedded subcutaneously [3-5]. After the surgical site had been rinsed with hydrogen peroxide and normal saline, a silica gel drainage strip was inserted and the tissues closed layer by layer. The surgery was completed by wrapping the elbow with sterile gauze.

\subsubsection{Plaster External Fixation}

Patients in the intervention group received plaster external fixation with the elbow at maximum extension immediately following surgery. Starting on day 1 post-surgery, the functional cast was adjusted daily to the maximum tolerable elbow flexion at 10am and then to the maximum tolerable elbow extension at $10 \mathrm{pm}$ for a duration of 3 weeks. Adjustment of the cast was performed by medical staff during hospitalization, and the procedure explained to the patients' families in order that the adjustments were performed at the appropriate time and angle after patient discharge. Patients in the control group were provided plaster fixation at an elbow flexion of $70-90^{\circ}$ post-surgery. Casts were removed from both groups at 3 weeks post-surgery and functional exercises initiated.

\subsection{Follow-up and Assessment}

Patients were followed up regularly by X-ray examination to assess bone healing and potential complications. Range of motion (ROM) measurements and modified Hospital for Special Surgery (HSS) elbow functional assessments were conducted at 1 and 3 months post-surgery. Images of a patient in each group are shown in Figures 1 and 2. 

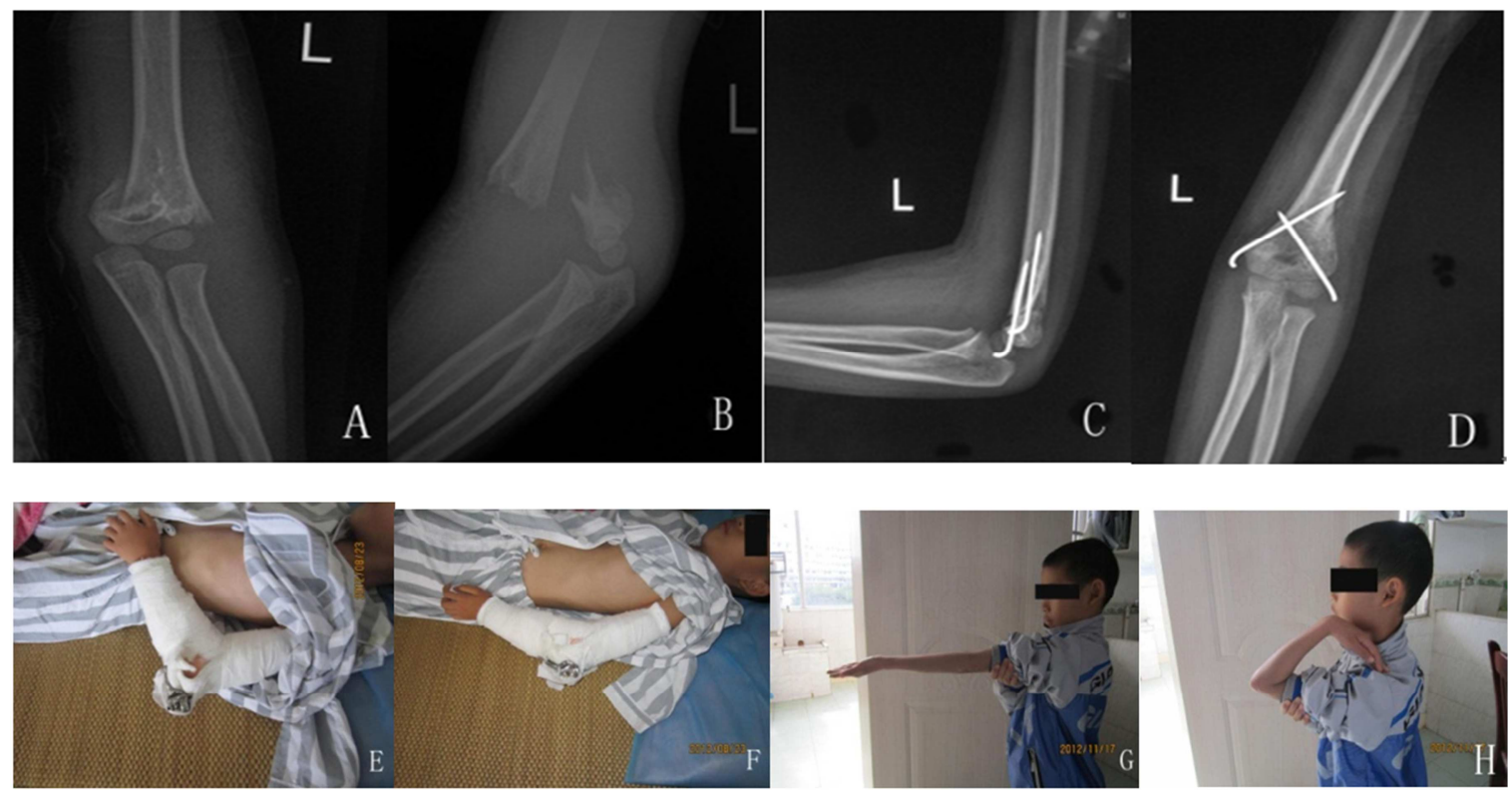

Figure 1. A patient in the intervention group diagnosed with left Gartland type III SHF. X-ray images of patient's elbow before (A and B) and after surgery (C and D). Patient was given post-surgery FTFAEF ( $E$ and F). Elbow function was assessed at 2 months post-surgery ( $G$ and $H$ ).
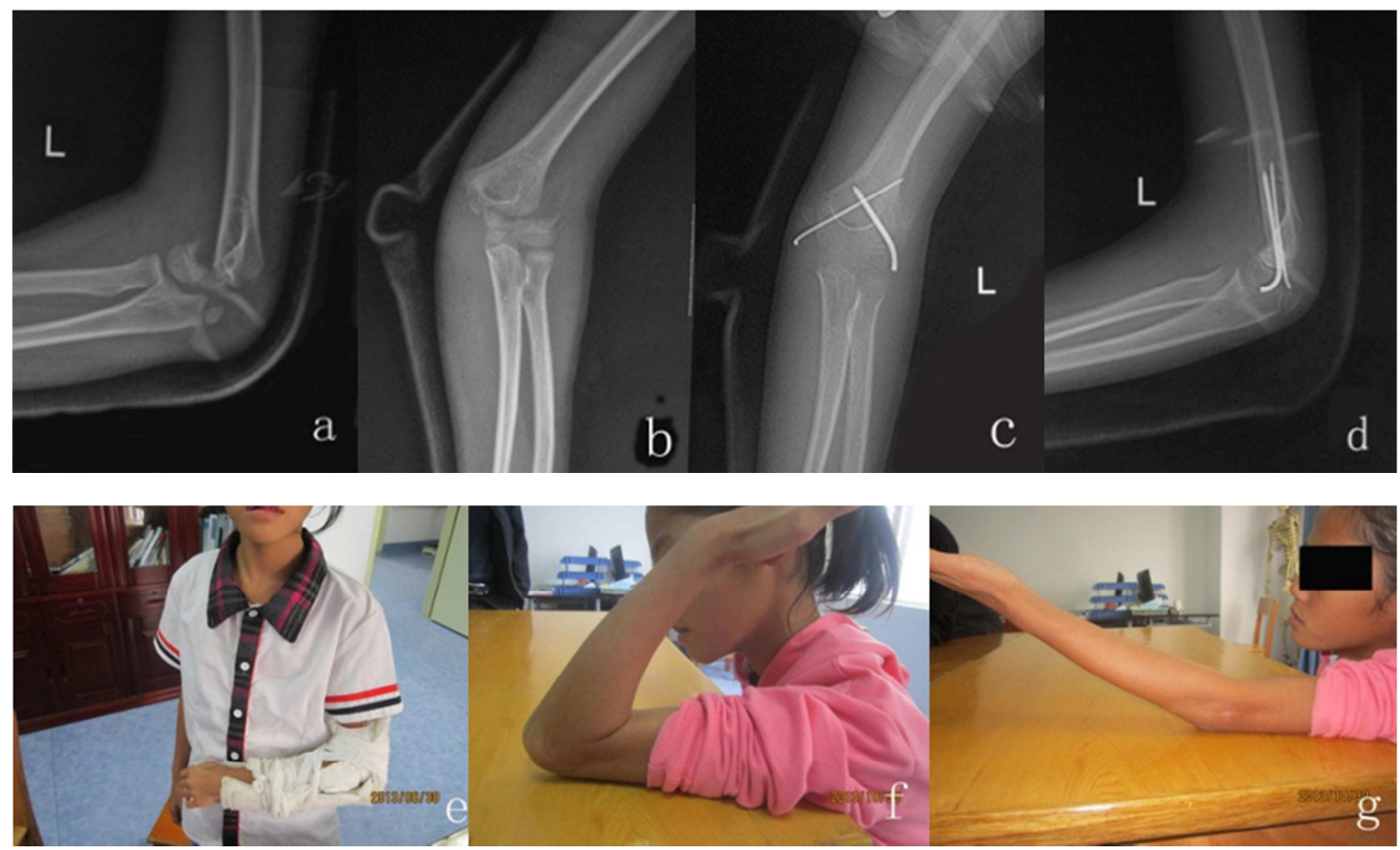

Figure 2. A patient in the control group diagnosed with left Gartland type III SHF. X-ray images of patient's elbow before (a and b) and after surgery (c and d). The patient was given conventional post-surgery plaster external fixation (e). Elbow function was assessed at 4 months post-surgery (f and g).

\subsection{Statistical Analysis}

Data were analyzed using SPSS version 16.0 software. Measured data, such as age, duration of bone healing or total HSS score were expressed as mean \pm SD $(\overline{\mathrm{X}} \pm \mathrm{S})$

and compared between the two groups using a two-sample t-test. Count data, such as complication and excellent HSS score rates, were compared between the two groups using a chi-square $\left(\mathrm{X}^{2}\right)$ test. $\mathrm{P}<0.05$ was considered statistically significant.

\section{Results}

Both intervention and control groups were followed up for a mean of 3.6 months (3-4 months). There was no significant difference in the duration of bone healing between the groups (7.6 \pm 1.5 versus $7.5 \pm 1.7$ weeks for the intervention and control groups, respectively, $\mathrm{P}>0.05$ ). No incidence of $\mathrm{K}$ needle loosening or atopic ossification was observed in any patient. At 1 month post-surgery, ROM and modified HSS 
scores were significantly higher in the intervention group $\left(85.8 \pm 6.1^{\circ}\right.$ and $65.2 \pm 3.6^{\circ}$, respectively) than in the control group (62.3 $\pm 5.2^{\circ}$ and $56.6 \pm 2.1^{\circ}$, respectively) (both $\mathrm{P}<0.05$; Tables 1 and 2). At 3 months post-surgery, ROM and modified HSS scores were again significantly higher in the intervention group $\left(132.0 \pm 4.7^{\circ}\right.$ and $98.5 \pm 1.3^{\circ}$, respectively) than in the control group $\left(107.5 \pm 24.4^{\circ}\right.$ and $85.0 \pm 10.3^{\circ}$, respectively) (both $\mathrm{P}<0.05$; Tables 3 and 4). Furthermore, excellence rate was significantly higher in the intervention group $(100 \%)$ than in the control group $(74.42 \%)(\mathrm{P}<0.05)$.

Table 1. Comparison of ROM measurements between the intervention and control groups 1 month post-surgery $(\bar{X} \pm S)$.

\begin{tabular}{|c|c|c|c|c|}
\hline Group & Patients Number(n) & Extension contracture $\left(^{\circ}\right)$ & Flexion contracture $\left({ }^{\circ}\right)$ & $\operatorname{ROM}\left({ }^{\circ}\right)$ \\
\hline Intervention & 86 & $23.1 \pm 3.5$ & $25.9 \pm 3.0$ & $85.8 \pm 6.1$ \\
\hline Control & 86 & $36.5 \pm 1.9$ & $36.9 \pm 3.8$ & $62.3 \pm 5.2$ \\
\hline $\mathrm{T}$ value & & -39.745 & -23.238 & 32.342 \\
\hline $\mathrm{P}$ value & & 0.000 & 0.000 & 0.009 \\
\hline
\end{tabular}

Table 2. Comparison of modified HSS scores of the intervention and control groups 1 month post-surgery.

\begin{tabular}{|c|c|c|c|c|c|c|c|c|}
\hline Group & Patients Number(n) & Total score & Excellent (n) & Good (n) & Average (n) & Bad (n) & Poor (n) & Excellence rate (\%) \\
\hline 1 & 86 & $65.2 \pm 3.6$ & 0 & 0 & 18 & 68 & 0 & 0 \\
\hline 2 & 86 & $56.6 \pm 2.1$ & 0 & 0 & 6 & 80 & 0 & 0 \\
\hline $\begin{array}{l}3 \\
P\end{array}$ & & $\mathrm{t}=24.299$ & & & & & & $\begin{array}{l}X 2=20.104 \\
0.000\end{array}$ \\
\hline
\end{tabular}

1: Intervention group; 2: control group; 3 :statistics

Table 3. Comparison of ROM measurements between the intervention and control groups 3 months post-surgery $(\bar{X} \pm S)$.

\begin{tabular}{llll}
\hline Group & Patients Number(n) & Extension contracture $\left({ }^{\circ}\right)$ & Flexion contracture $\left({ }^{\circ}\right)$ \\
\hline Intervention & 86 & $1.7 \pm 2.413 .8 \pm 15.8-$ & $1.7 \pm 3.0$ \\
Control & 86 & 7.061 & $11.1 \pm 4.4-17.575$ \\
T value & & 0.001 & 0.001 \\
P value & & & $132.0 \pm 4.7$ \\
\hline
\end{tabular}

Table 4. Comparison of modified HSS scores between the intervention and control.

\begin{tabular}{|c|c|c|c|c|c|c|c|c|}
\hline Group & Patients Number(n) & Total score & Excellent (n) & $\operatorname{Good}(n)$ & Average (n) & Bad (n) & Poor (n) & Excellence rate $(\%)$ \\
\hline 1 & 86 & $98.5 \pm 1.3$ & 86 & 0 & 0 & 0 & 0 & 100 \\
\hline 2 & 86 & $85.0 \pm 10.3$ & 30 & 34 & 18 & 1 & 3 & 74.42 \\
\hline 3 & & $\mathrm{t}=12.146$ & & & & & & $X^{2}=25.227$ \\
\hline$P$ & & & & & & & & 0.000 \\
\hline
\end{tabular}

\section{Discussion}

SHFs occur at the boundary of the humeral shaft and condyle. The Gartland classification system categorizes SHF into 3 categories based on severity of displacement. Specifically, type I SHF is not displaced; type II is angulated with the posterior cortex intact; type III is completely displaced with no cortical contact[6,7]. Bone displacement in Gartland type III SHF is difficult to correct by manipulative reduction as the realigned fractured ends have minimal contact and poor stability, making splint and plaster external fixation extremely difficult. As a result, bone displacement may reoccur and lead to deformed humeral healing. Therefore, surgery is generally used in the treatment of Gartland type III SHF[8-10]. As closed reduction with percutaneous fixation has become more popular, many children with Gartland type III SHF have achieved satisfactory bone realignment and relatively stable fixation. However, ORIF was still required for a small number of patients in these large case studies. In fact, open reduction and crossed $\mathrm{K}$-needle fixation is still the primary treatment for children with Gartland type III SHF in many county hospitals. This approach involves an incision lateral to the humerous and crossed K-needle internal fixation, which not only avoids injury to the triceps but also maintains integrity of the elbow extension device. Furthermore, this method allows direct view of the reduction and complete postsurgery drainage, with reduced risk of atopic ossification. Fixation is achieved by insertion of the K-needles from both sides of the proximal end towards the cortical bone of the opposite end The crossing technique can provide good stability to the fractured ends and reduce surgical injury to a minimum.

Elbow dysfunction is a common complication of SHF and is primarily caused by soft tissue damage [9-13]. Elbow injury leads to local hematoma formation, and the biological response to the reaction of the blood can cause the joint capsule to contract[11-14]. In addition, absorption of the blood and exudate often results in scar tissue formation and hyperplasia, and may even lead to elbow stiffness in some serious cases[15-17].

Soft tissue has both elastic and plastic properties [18-20]. Elastic deformation occurs when periarticular soft tissue stretches in response to the stress generated by elbow movement, returning to its original length upon removal of the stress. On the other hand, plastic deformation occurs when stretched soft tissue maintains its stretched length even 
after removal of the stress. Whether soft tissues undergo elastic or plastic deformation is dependent on the magnitude and duration of the force.

The anterior and posterior elbow joint capsules are of a length that allows them to fold and expand as the elbow flexes and extends. The anterior joint capsule is maximally expanded when the patient's elbow is fixed at its maximum extension by the functional cast. The tension generated by this movement not only causes elastic deformation of scar tissues within the anterior side of the elbow, countering anterior joint capsule contracture, it also promotes the anterior joint capsule to form a "repair template" on which the scar tissues can be maintained in their most stretched form. Meanwhile, elbow extension causes the posterior joint capsule to adopt its shortest and most folded form. This position can easily lead to contracture of the posterior joint capsule and formation of scar tissue, causing any freshly generated scar tissue around the posterior joint capsule to be in their most contracted form. When the elbow is fixed at maximum extension for a long period of time, the posterior joint capsule contracts and hypertrophic scar tissues eventually reach a steady state such that subsequent flexion creates substantial tension that prevents the elbow from bending. Therefore, once the elbow has been fixed at maximum extension for 12 hours, it should be bent and fixed at maximum flexion to allow the posterior joint capsule to stretch out into its maximally expanded form. This tension causes the scar tissues and contracted posterior joint capsule to undergo elastic deformation that counters the posterior joint capsule contraction. In addition, the posterior joint capsule forms a "repair template" on which the hypertrophic scar tissues can be maintained in their most stretched out form. Similarly, when the elbow is fixed at maximum flexion for a long period of time, the anterior joint capsule contracts and hypertrophic scar tissues eventually reach a steady state, such that subsequent extension can create substantial tension that prevents the elbow from extending. Therefore, once the elbow has been fixed at maximum flexion for 12 hours, it should be extended and fixed at maximum extension to allow the anterior joint capsule and scar tissues to repair in their most stretched out form.

Conventional plaster external fixation for Gartland type III SHF fixes the elbow at $70-90^{\circ}$ for 3 weeks post-surgery. When the elbow is fixed in such a manner, both the anterior and posterior joint capsules and the lateral collateral ligaments (LCLs) are in their most relaxed form. Long-term fixation at this position can easily lead to contractures of these components causing elbow dysfunction.

In this study, patients' elbows were fixed at maximum flexion at 10am to facilitate daily activities and then at maximum extension at $10 \mathrm{pm}$ to aid sleeping. Twelve hours of sustained stress can promote elastically deformed soft tissues to undergo further plastic deformation, allowing the anterior and posterior humeral soft tissues to reach their maximum lengths at steady state and prevent tension during elbow flexion and extension. We found that 3 weeks of FTFAEF, during which the anterior and posterior joint capsules and hypertrophic scar tissues undergo repair cycles of "anterior extended posterior contracted" to "anterior contracted posterior extended", resulted in satisfactory elbow activity shortly after surgery and provided a good foundation for subsequent rehabilitation of elbow functions. ROM, modified HSS scores, and the number of excellent ratings at 3 months post-surgery were significantly higher in the intervention group $\left(132.0 \pm 4.7^{\circ}, 98.5 \pm 1.3^{\circ}\right.$ and $100 \%$, respectively) than in the control group $\left(107.5 \pm 24.4^{\circ}, 85.0 \pm 10.3^{\circ}\right.$ and $74.42 \%$, respectively) (all $\mathrm{P}<0.05)$. Conversely, there was no significant difference in the duration of clinical bone fracture healing between the two groups $(\mathrm{P}>0.05)$ and no incidence of $\mathrm{K}$-needle loosening or atopic ossification in either group, demonstrating that FTFAEF does not increase the risk of complications.

\section{Conclusion}

In conclusion, elbow organized hematoma and soft tissue contracture are the major causes of elbow-joint dysfunction following an operation of the Gartland type III supracondylar humerus fractures.

The characteristics of soft tissue are elasticity and plasticity. Whether the soft tissue presents elastic deformation or plastic deformation depends on the magnitude and duration of the stress. In this study, Twelve hours of sustained stress can promote elastically deformed soft tissues to undergo further plastic deformation, allowing the anterior and posterior humeral soft tissues to reach their maximum lengths at steady state and prevent tension during elbow flexion and extension.

We found that 3 weeks of fixed-time and fixed-angle external fixation resulted in satisfactory elbow activity shortly after surgery and provided a good foundation for subsequent rehabilitation of elbow functions.And FTFAEF does not increase the risk of complications.

\section{References}

[1] Sharma A, Walia JP, Brar BS, Sethi S. Early results of displaced supracondylar fractures of humerus in children treated byclosed reduction and percutaneous pinning $[\mathrm{J}]$. Indian J Orthop. 2015 Sep-Oct;49(5):529-35.

[2] Fan JR, Xu YW, Zheng Y, You JY. Emergency closed reduction and percutaneous Kirschner wire fixation for treatment of Gartland type II-III supracondylar fractures of the humerus in children. [J]. Zhongguo Gu Shang. 2015 May;28(5):464-7.

[3] Guven MF, Kaynak G, Inan M, Caliskan G, Unlu HB, Kesmezacar H. Results of displaced supracondylar humerus fractures treated with open reduction and internal fixation after a mean 22.4 years of follow-up[J]. J Shoulder Elbow Surg. 2015 Apr;24(4):640-6.

[4] [4]Hussain S,Ahmad M,Muzaffar T. Open reduction and internal fixation for displaced supracondylar fractures of the humerus in children with crossed K-wires via lateral approach[J].Chin J Traumatol。2014; 17(3):130-5. 
[5] Ahmad Khan RDO, Yousaf MN, Zain-Ur-Rehman M, Yasin A. Utcome of open reduction internal fixation with cross Kwires for supracondylar fracture of humerus in terms of Flynn's criteria in children[J]. J Pak Med Assoc.2015 Nov ;65(11 Suppl 3): S186-9.

[6] [6]Gartland JJ.Management of supracondylar fractures of the humerus in children[J].Surg Gynecol Obstet,1959,109:145154

[7] Rocha IT,Faria Ade S,Filho CF,Rocha, MA.Reproducibility of the AO/ASIF and Gartland classifications for supracondylar fractures of the humerus in children[J].Rev Bras Ortop.2015;50(3) :266-9.

[8] Sarrafan N, Nasab SA, Ghalami T. Treatment of displaced supracondylar fracture of the humerus in children by open pining from lateral approach: an investigation of clinical and radiographical results[J]. Pak J Med Sci. 2015 JulAug;31(4):930-935.

[9] Ahmad Khan, RD; Yousaf, MN; Zain - Ur Rehman, M; Yasin, A; Etc. Outcome of open reduction internal fixation with cross $\mathrm{K}$-wires for supracondylar fracture of humerus in terms of Flynn's criteria in children[J].J Pak Med Assoc.2015 Nov;65(11 Suppl 3): S186-189.

[10] Dhoju D, Shrestha D, Parajuli N, Dhakal G, Shrestha R. Displaced supracondylar humeral fractures in children: Comparison of three treatment approaches[J]. Kathmandu Univ Med J (KUMJ). 2011 Apr-Jun;9(34):11-16.

[11] Kopka M, Monument MJ, Befus AD, Hildebrand KA.Serum Mast Cell Tryptase as a Marker of Posttraumatic Joint Contracture in a Rabbit Model[J]. J Orthop Trauma.2017 Mar ;31(3): e86-e89.

[12] Sun Yangbai, Li Fengfeng, Fan Cunyi.Effect of pERK2 on extracellular matrix turnover of the fibrotic joint capsule in a post-traumatic joint contracture model[J]. Exp Ther Med.2016 Feb ;11(2) 547-552.

[13] Hildebrand KA, Zhang M, Hart DA. Myofibroblast upregulators are elevated in joint capsules in posttraumatic contractures[J]. Clin Orthop Relat Res. 2007 Mar; 456:85-91.

[14] Hildebrand KA, Zhang M, Germscheid NM, Wang C, Hart DA. Cellular, matrix, and growth factor components of the joint capsule are modified early in the process of posttraumatic contracture formation in a rabbit model[J]. Acta Orthop. 2008 Feb;79(1):116-25.

[15] Michelsson JE, Rauschning W. Pathogenesis of experimental heterotopic bone formation following temporary forcible exercising of immobilized limbs[J]. Clin Orthop,1983,17(6):265-272.

[16] Hotchkiss RN. Elbow contracture/Green DP, Hotchkiss RN, Pederson WC, Wolfe SW(eds). Green's Operative Hand Surgery[M]. 5thed.New York, NY: Church illLivingstone, 2005:667-682.

[17] Wu, Xinghuo; Wang, Hong; Meng, Chunqing; Etc.Outcomes of arthroscopic arthrolysis for the post-traumatic elbow stiffness[J]. Knee Surg Sports Traumatol Arthrosc.2015;23(9):2715-20.

[18] Pradas MM, Calleja RD.Nonlinear viscoilastec behaviour of the flexor tendon of the human hand [J].J Biomechan, 1990,23:773.

[19] Spirt AA,Mak AF,Wassell RP.Nonlinear viscoelastic properties of articular cartilage in shear[J].J Orthop Res, 1989,7:3.

[20] Taylor DC,Dalton JD.Viscoelastic properties of muscle tendon units:The biomechanical effects of stretching $[\mathrm{J}]$.Am J Sport Med,1990,18:300. 